\title{
Spin dynamics in the generalized ferromagnetic Kondo model for manganites.
}

\author{
N.B.Perkins and N.M.Plakida \\ Joint Institute for Nuclear Research, Dubna 141980, Russia
}

\begin{abstract}
Dynamical spin susceptibility is calculated for the generalized ferromagnetic Kondo model which describes itinerant $e_{g}$ electrons interacting with localized $t_{2 g}$ electrons with antiferromagnetic coupling. The calculations done in the mean field approximation show that the spin-wave spectrum of the system in ferromagnetic state has two branches, acoustic and optic ones. Self-energy corrections to the spectrum are considered and the acoustic spin-wave damping is evaluated.
\end{abstract}




\section{Introduction}

Manganites of the perovskite structure of the form $R_{1-x} B_{x} \mathrm{MnO}_{3}$ (where $R$ are trivalent rare-earth and $B$ are divalent alcaline ions, respectively) and related compounds that present the phenomenon of "colossal" magnetoresistance (CMR) have recently attracted much attention both from the basic point of view and due to their potential application [1], [2]. The large magnetoresistance occurs close to the metal-insulator and the paramagnetic-ferromagnetic transitions where the interplay of transport, magnetic and structural properties is of the great importance (see [3]). The key elements of the manganese oxides are $\mathrm{Mn}$ ions. In the parent compound $\mathrm{LaMnO}_{3}$ the electronic configuration of $\mathrm{Mn}^{+3}$ is $\left(t_{2 g}^{3} e_{g}\right)$. In this configuration due to a strong intra-orbital Hund's coupling $t_{2 g}^{3}$ electrons go into tightly bound $d_{x y}, d_{y z}, d_{x z}$ core states and make up an electrically inert core Heisenberg spins $S$ of magnitude 3/2. The $t_{2 g}^{3}$ configuration is very stable and remains localized over the entire range of doping.

In the undoped case, with one $e_{g}$ electron per $M n$ ion, two $e_{g}$ orbitals, $d_{x^{2}-y^{2}}$ and $d_{3 z^{2}-r^{2}}$ types, are splitted due to the Jahn-Teller effect. At low temperature $e_{g}$ electrons occupy $d_{3 x^{2}-r^{2}}$ and $d_{3 y^{2}-r^{2}}$ ordered alternately in $a b$ plane with their spins aligned to the core spin by interorbital Hund's coupling. Due to the GoodenoughKanamori rules [4] it results in the A-type antiferromagnet (AFM) ground state (AFM vector $\mathrm{Q}=(0,0,0.5))$ with spin $S=2$ for $\mathrm{LaMnO}_{3}$. Upon doping with holes by substituting $L a$ with $S r$ or any other divalent ions system becomes ferromagnetic (FM) and conducting. The hopping between two $M n$ sites is maximal when the core spins are parallel and minimum when they are antiparallel.That results in effective ferromagnetic exchange between the nearest neighbor core spins and thus leads to the FM metallic ground state of doped compounds. This behavior is qualitatively well described within the framework of the double exchange (DE) mechanism (see [5], [6], [0] ). At higher hole concentration, $x \geq 0.5$, a charge ordering for holes is observed, and at $x=1$ an insulating G-type AFM state (with $Q=(0.5,0.5,0.5)$ ) takes place for $\mathrm{CaMnO}_{3}$ compound. Therefore to describe the experimentally obtained phase diagram ( see, for example, [8]) one should take into account both the Heisenberg type of AFM exchange between the core $t_{2 g}$ electrons and the strong Hund coupling between $t_{2 g}$ and $e_{g}$ electrons (see, e.g. [9, 10]). These competing interactions could be responsible for a coexistence of AFM and FM states observed recently in neutron scattering experiments in $\left(\mathrm{La}_{0.25} \mathrm{Pr}_{0.75}\right)_{0.7} \mathrm{Ca}_{0.3} \mathrm{MnO}_{3}$ [11] and in the bilayer manganite $\mathrm{La}_{1.2} \mathrm{Sr}_{1.8} \mathrm{Mn}_{2} \mathrm{O}_{7}$ [12]. Also a crossover from an ideal isotropic FM spin-wave behaviour at low temperature to a diffusive spin propagation observed in $\mathrm{La}_{0.7} \mathrm{Ca}_{0.3} \mathrm{MnO}_{3}$ [13] could be explained if one takes into account both the localized $t_{2 g}$ spin $(S=3 / 2)$ and the itinerant $e_{g}$ spin $(\sigma=1 / 2)$ subsystems.

In the present paper we study the spin dynamics in manganites within the generalized ferromegnetic Kondo model (FKM) allowing for the AFM exchange interaction between $t_{2 g}$ spins. Unlike to the DE model (see, [14]), where $J_{H} / t \rightarrow \infty$ is considered and the system is treated as perfectly spin polarized with $S=2$, in our work both the fluctuation of the localized and itinerant spins are taking into account. However, we ignore in the present calculations a possible orbital ordering 
which is very important in explaining different types of AFM ordering in the insulating phases [10, 15 but plays less essential role in the FM state considered here. To take into account strong Coulomb interaction between $e_{g}$ electrons which excludes the double occupancy of $e_{g}$ electrons for a lattice site we employ the Hubbard operator technique. The spectrum of spin waves in the FM state is calculated by employing equations of motion for the matrix Green function (GF) for the localized and itinerant spins. In the next Section the model and general formalism for the GF are presented. The spin-wave spectrum in a generalized mean field approximation (MFA) is calculated in Sec. 3 and self-energy corrections and spin-wave damping are evaluated in Sec. 4.

\section{The model}

We consider an effective Hamiltonian of the generalized FM Kondo model which can be written in the following form [9]:

$$
H=-\sum_{i, j, \sigma} t_{i j} X_{i}^{\sigma 0} X_{j}^{0 \sigma}-\frac{J_{H}}{2 S} \sum_{i} \mathbf{S}_{i} \sigma_{i}+\frac{1}{2} \sum_{i, j} J_{i j} \mathbf{S}_{i} \mathbf{S}_{j}
$$

The first term of Eq. (11) describes an electron hopping between Mn-ions where $X_{i}^{\sigma 0}$ is the creation operator of an electron with spin $\sigma$ in one of the $e_{g}$ orbitals. Here we neglect orbital degeneracy of $e_{g}$ electrons and introduce orbital independent hopping parameter $t_{i j}$ with $t_{i j}=t$ for the nearest neighbors. The second term describes the ferromagnetic Hund coupling $\left(J_{H}>0\right)$ between $e_{g}$ and $t_{2 g}$ spins where $\mathbf{S}_{i}$ refers to the localized Mn core spin $S=3 / 2$. The third term describes the antiferromagnetic coupling of localized spins between the nearest neighbor sites. In real materials the coupling of core spins is not the same in different directions and should be described in the matrix form, but for simplicity we are analyzing the isotropic case $\left(J_{i j}=J\right)$. We exclude the doubly occupied $e_{g}$ state from the effective Hamiltonian by using the Hubbard operator representation because the electron-electron interaction has the largest energy scale (intra-atomic Coulomb interaction in the $e_{g}$ orbitals) and can be estimated as $7-8 \mathrm{eV}$ while $J_{H} \sim 1 \mathrm{eV}$. Due to large Hund energy we neglect superexchange interaction between $e_{g}$ electrons of the order of $t^{2} / U$ [10. The conduction bandwidth is smaller than the Hund coupling energy and from density-functional studies can be estimated as $t \simeq 0.15 \mathrm{eV}$ [16].

The HO's in Eq. (11) are defined as $X_{i}^{\alpha \beta}=|i, \alpha\rangle\langle i, \beta|$ for three possible states at the lattice site $i:|i, \alpha\rangle=|i, 0\rangle, \quad|i, \sigma\rangle$ for an empty site and for a singly occupied site with spin $\sigma=(\uparrow, \downarrow)=(+,-)$. The completeness relation for the HO's reads as

$$
X_{i}^{00}+\sum_{\sigma} X_{i}^{\sigma \sigma}=1
$$

For itinerant electrons the spin and density operators in Eq. (11) are expressed by HO's as

$$
\sigma_{i}^{+}=X_{i}^{\uparrow \downarrow}, \sigma_{i}^{-}=X_{i}^{\downarrow \uparrow}, \sigma_{i}^{z}=\frac{1}{2}\left(X_{i}^{\uparrow \uparrow}-X_{i}^{\downarrow \downarrow}\right), \quad n_{i}=X_{i}^{\uparrow \uparrow}+X_{i}^{\downarrow \downarrow}
$$


The HO's obey the following commutation relations

$$
\left[X_{i}^{\alpha \beta}, X_{j}^{\gamma \delta}\right]_{ \pm}=\delta_{i j}\left(\delta_{\beta \gamma} X_{i}^{\alpha \delta} \pm \delta_{\delta \alpha} X_{i}^{\gamma \beta}\right)
$$

In Eq.(四) the upper sign stands for the case when both HO's are Fermi-like ones (as, e. g., $X_{i}^{0 \sigma}$ ). The spin and density operators (3) are Bose-like and for them the lower sign in Eq.(4) should be taken.

It is assumed that the core spin operators $S_{i}^{\alpha}$ obey the standard commutation relations, e.g.,

$$
\left[S_{i}^{+}, S_{j}^{-}\right]=2 \delta_{i, j} S_{j}^{z}
$$

To treat the fluctuations of localized and itinerant spins at the same level of approximation we introduce the dynamic spin susceptibility (DSS) of the system in the matrix form

$$
\chi(q, \omega)=\left(\begin{array}{ll}
\chi_{11} & \chi_{12} \\
\chi_{21} & \chi_{22}
\end{array}\right)=\left\langle\left\langle A_{q} \mid A_{q}^{+}\right\rangle\right\rangle_{\omega}
$$

where

$$
A_{q}=\left(\begin{array}{c}
\sigma_{q}^{+} \\
S_{q}^{+}
\end{array}\right), \quad A_{q}^{+}=\left(\begin{array}{ll}
\sigma_{q}^{-} & S_{q}^{-}
\end{array}\right)
$$

Here

$$
\left\langle\left\langle A_{q} \mid A_{q}^{+}\right\rangle\right\rangle_{\omega}=-\imath \int_{0}^{\infty} d t e^{-\imath \omega t} \frac{1}{N} \sum_{q} e^{-\imath q(l-m)}\left\langle\left[A_{l}(t), A_{m}^{+}\right]\right\rangle
$$

denotes the Fourier transformed two-time retarded commutator Green function (GF) 18, 19. The diagonal elements $\chi_{11}(q, \omega)$ and $\chi_{22}(q, \omega)$ stands for the itinerant and core spin GF, respectively, while the nondiagonal elements $\chi_{12}(q, \omega)$ and $\chi_{21}(q, \omega)$ define the crosscorrelations between the two spin subsystems. The GF (6) obeys the following equation of motion

$$
\begin{array}{r}
\omega\left\langle\left\langle A_{q} \mid A_{q}^{+}\right\rangle\right\rangle_{\omega}=\left\langle\left[A_{q}, A_{q}^{+}\right]\right\rangle+\left\langle\left\langle\imath \dot{A}_{q} \mid A_{q}^{+}\right\rangle\right\rangle_{\omega}, \\
\omega\left\langle\left\langle\imath \dot{A}_{q} \mid A_{q}^{+}\right\rangle\right\rangle_{\omega}=\left\langle\left[\imath \dot{A}_{q}, A_{q}^{+}\right]\right\rangle+\left\langle\left\langle\imath \dot{A}_{q} \mid-\imath \dot{A}_{q}^{+}\right\rangle\right\rangle_{\omega} .
\end{array}
$$

These equations (8) could be easily combined in a more convenient form of the equation of motion $[19$ :

$$
\begin{gathered}
\omega\left\langle\left\langle A_{q} \mid A_{q}^{+}\right\rangle\right\rangle_{\omega}=\left\langle\left[A_{q}, A_{q}^{+}\right]\right\rangle \\
+\left(\left\langle\left[\imath \dot{A}_{q}, A_{q}^{+}\right]\right\rangle+\left\langle\left\langle\imath \dot{A}_{q} \mid-\imath \dot{A}_{q}^{+}\right\rangle\right\rangle_{\omega}^{i r r}\right) \cdot \frac{1}{\left\langle\left[A_{q}, A_{q}^{+}\right]\right\rangle} \cdot\left\langle\left\langle A_{q} \mid A_{q}^{+}\right\rangle\right\rangle_{\omega},
\end{gathered}
$$

where the current is defined as $\imath \dot{A}=\imath d A / d t=[A, H]$ and in the matrix form can be given by the following expression:

$$
\imath \dot{A}_{q}=\left(\begin{array}{c}
\imath \dot{\sigma}_{q}^{+} \\
\imath \dot{S}_{q}^{+}
\end{array}\right)
$$

and

$$
\left\langle\left\langle\imath \dot{A}_{q} \mid-\imath \dot{A}_{q}^{+}\right\rangle\right\rangle_{\omega}^{i r r}=\left\langle\left\langle\imath \dot{A}_{q} \mid-\imath \dot{A}_{q}^{+}\right\rangle\right\rangle_{\omega}
$$




$$
\text { - }\left\langle\left\langle\imath \dot{A}_{q} \mid A_{q}^{+}\right\rangle\right\rangle_{\omega}\left\langle\left\langle A_{q} \mid A_{q}^{+}\right\rangle\right\rangle_{\omega}^{-1}\left\langle\left\langle A_{q} \mid-\imath \dot{A_{q}^{+}}\right\rangle\right\rangle_{\omega}
$$

is the irreducible part of the higher order GF.

We can rewrite (9) in the Dyson form

$$
\chi_{q}(\omega)=\left[\omega \hat{\tau}_{0}-\tilde{\Omega}_{q}-\tilde{\Pi}(q, \omega)\right]^{-1} \cdot I,
$$

where $\hat{\tau}_{0}$ is the unity matrix and

$$
I=\left\langle\left[A_{q}, A_{q}^{+}\right]\right\rangle=\left(\begin{array}{cc}
\left\langle\left[\sigma_{q}^{+}, \sigma_{q}^{-}\right]\right\rangle & \left\langle\left[\sigma_{q}^{+}, S_{q}^{-}\right]\right\rangle \\
\left\langle\left[S_{q}^{+}, \sigma_{q}^{-}\right]\right\rangle & \left\langle\left[S_{q}^{+}, S_{q}^{-}\right]\right\rangle
\end{array}\right)=\left(\begin{array}{cc}
2\left\langle\sigma^{z}\right\rangle & 0 \\
0 & 2\left\langle S^{z}\right\rangle
\end{array}\right)
$$

where $\left\langle\sigma^{z}\right\rangle=\left\langle\sigma_{l}^{z}\right\rangle$ and $\left\langle S^{z}\right\rangle=\left\langle S_{l}^{z}\right\rangle$.

The matrix $\tilde{\Omega}_{q}=\Omega_{q} I^{-1}$ describes the mean field (MF) energy spectrum and $\tilde{\Pi}(q, \omega)=\Pi(q, \omega) I^{-1}$ is the self-energy matrix. They are given by

$$
\begin{aligned}
& \Omega_{q}=\left\langle\left[\imath \dot{A}_{q}, A_{q}^{+}\right]\right\rangle=\left(\begin{array}{cc}
\left\langle\left[\imath \dot{\sigma}_{q}^{+}, \sigma_{q}^{-}\right]\right\rangle & \left\langle\left[\dot{\sigma}_{q}^{+}, S_{q}^{-}\right]\right\rangle \\
\left\langle\left[\imath \dot{S}_{q}^{+}, \sigma_{q}^{-}\right]\right\rangle & \left\langle\left[\imath \dot{S}_{q}^{+}, S_{q}^{-}\right]\right\rangle
\end{array}\right), \\
& \Pi(q, \omega)=\left\langle\left\langle\imath \dot{A}_{q} \mid-\imath \dot{A_{q}^{+}}\right\rangle\right\rangle^{i r r}=\left(\begin{array}{ll}
\left.\left\langle\left\langle\imath \dot{\sigma}_{q}^{+} \mid \imath \sigma_{q}^{-}\right\rangle\right\rangle\right\rangle^{i r r} & \left\langle\left\langle\imath \dot{\sigma}_{q}^{+} \mid \imath \dot{S}_{q}^{-}\right\rangle\right\rangle^{i r r} \\
\left\langle\left\langle\imath \dot{S}_{q}^{+} \mid \imath \sigma_{q}^{-}\right\rangle\right\rangle^{i r r} & \left\langle\left\langle\imath \dot{S}_{q}^{+} \mid \imath S_{q}^{-}\right\rangle\right\rangle^{i r r}
\end{array}\right),
\end{aligned}
$$

with

$$
\begin{gathered}
\dot{\sigma_{l}^{+}}=\sum_{i} t_{i l}\left(X_{i}^{\uparrow 0} X_{l}^{0 \downarrow}-X_{l}^{\uparrow 0} X_{i}^{0 \downarrow}\right)-\frac{J_{H}}{2 S}\left(S_{l}^{+} \sigma_{l}^{z}-S_{l}^{z} \sigma_{l}^{+}\right), \\
\imath \dot{S_{l}^{+}}=-\frac{J_{H}}{2 S}\left(S_{l}^{z} \sigma_{l}^{+}-S_{l}^{+} \sigma_{l}^{z}\right)-\sum_{i} J_{i l}\left(S_{i}^{z} S_{l}^{+}-S_{l}^{z} S_{i}^{+}\right) .
\end{gathered}
$$

\section{Mean field approximation}

Let us now examine the spectrum and DSS in mean field approximation (MFA). The spin-wave dispersion is determined by the following equation

$$
\operatorname{det}\left(\omega \hat{\tau}_{0}-\tilde{\Omega}_{q}\right)=0 .
$$

From (14) we obtain for the matrix elements of $\tilde{\Omega}_{q}$

$$
\tilde{\Omega}_{q}=\left(\begin{array}{cc}
{\left[d+a\left(1-\gamma_{q}\right)\right] / 2\left\langle\sigma^{z}\right\rangle} & -d / 2\left\langle S^{z}\right\rangle \\
-d / 2\left\langle\sigma^{z}\right\rangle & {\left[d-b\left(1-\gamma_{q}\right)\right] / 2\left\langle S^{z}\right\rangle}
\end{array}\right),
$$

where we are using the following notation:

$$
\begin{aligned}
& d=\frac{J_{H}}{2 S}\left(2\left\langle\sigma_{l}^{z} S_{l}^{z}\right\rangle+\left\langle\sigma_{l}^{+} S_{l}^{-}\right\rangle\right), \\
& a=z t\left(n_{1}^{\uparrow}+n_{1}^{\downarrow}\right), \quad b=z J N_{1},
\end{aligned}
$$


with $t_{q}=z t \gamma_{q}, \gamma_{q}=(2 / z)\left(\cos q_{x}+\cos q_{y}+\cos q_{z}\right)$, where $z=6$ for the simple threedimensional cubic lattice with nearest-neighbor hopping $t$. In ( 21) the nearest neighbor particle-hole and spin correlation functions are defined as follows

$$
\begin{array}{r}
n_{1}^{\sigma}=\frac{1}{N} \sum_{k} \gamma_{k} n_{k}^{\sigma}, \quad n_{k}^{\sigma}=\left\langle X_{k}^{\sigma 0} X_{k}^{0 \sigma}\right\rangle \\
N_{1}=\frac{1}{N} \sum_{k} \gamma_{k} N_{k}, \quad N_{k}=2\left\langle S_{k}^{z} S_{-k}^{z}\right\rangle+\left\langle S_{k}^{+} S_{k}^{-}\right\rangle .
\end{array}
$$

The equation (18) has two solutions describing two branches of spin wave excitations:

$$
E_{q}^{1(2)}=\frac{1}{2}\left[\tilde{\Omega}_{q}^{11}+\tilde{\Omega}_{q}^{22} \mp \sqrt{\left(\tilde{\Omega}_{q}^{11}-\tilde{\Omega}_{q}^{22}\right)^{2}+4 \tilde{\Omega}_{q}^{12} \tilde{\Omega}_{q}^{21}}\right] .
$$

For the model calculation we can expand this equation at $q \rightarrow 0$ and for the finite value of $d$ we obtain

$$
\begin{array}{r}
E_{q}^{(1)} \simeq D_{1} q^{2} \\
E_{q}^{(2)} \simeq \Delta+D_{2} q^{2}
\end{array}
$$

where $E_{q}^{(1)}$ corresponds to the gapless (acoustic) spin-wave excitation with the stiffness $D_{1}$ given by

$$
D_{1}=\frac{a-b}{12\left(\left\langle S^{z}\right\rangle+\left\langle\sigma^{z}\right\rangle\right)},
$$

and $E_{q}^{(2)}$ describes the optic mode of the spin fluctuations with the gap $\Delta$ and the effective stiffness $D_{2}$ determined by the following expressions:

$$
\begin{array}{r}
\Delta=d \frac{\left\langle S^{z}\right\rangle+\left\langle\sigma^{z}\right\rangle}{2\left\langle S^{z}\right\rangle\left\langle\sigma^{z}\right\rangle}, \\
D_{2}=\frac{a\left\langle S^{z}\right\rangle^{2}-b\left\langle\sigma^{z}\right\rangle^{2}}{12\left\langle S^{z}\right\rangle\left\langle\sigma^{z}\right\rangle\left(\left\langle S^{z}\right\rangle+\left\langle\sigma^{z}\right\rangle\right)} .
\end{array}
$$

The ferromagnetic acoustic spin-wave becomes unstable when the stiffness $D_{1} \rightarrow 0$ or $a-b=0$ in Eq. (25). It may happen for small concentration of itinerant electrons, $n \leq n_{c} \simeq 2 S J / t \simeq 0.3$. The self-energy corrections considered below (see Eq. (47)) even increase the critical value $n_{c}$.

The spectrum of spin fluctuations in MFA are given by the spectral functions

$$
B_{\alpha \beta}^{M F}(q, \omega)=-\frac{1}{\pi} \operatorname{Im} \chi_{\alpha \beta}^{M F}(q, \omega+\imath \varepsilon)
$$

for the spin susceptibility

$$
B_{11}^{M F}(q, \omega)=2\left\langle\sigma^{z}\right\rangle\left(\frac{\tilde{\Omega}_{q}^{22}-E_{q}^{(1)}}{E_{q}^{(2)}-E_{q}^{(1)}} \delta\left(\omega-E_{q}^{(1)}\right)+\frac{E_{q}^{(2)}-\tilde{\Omega}_{q}^{22}}{E_{q}^{(2)}-E_{q}^{(1)}} \delta\left(\omega-E_{q}^{(2)}\right)\right)
$$




$$
\begin{gathered}
B_{22}^{M F}(q, \omega)=2\left\langle S^{z}\right\rangle\left(\frac{\tilde{\Omega}_{q}^{11}-E_{q}^{(1)}}{E_{q}^{(2)}-E_{q}^{(1)}} \delta\left(\omega-E_{q}^{(1)}\right)+\frac{E_{q}^{(2)}-\tilde{\Omega}_{q}^{11}}{E_{q}^{(2)}-E_{q}^{(1)}} \delta\left(\omega-E_{q}^{(2)}\right)\right), \\
B_{12}^{M F}(q, \omega)=B_{21}^{M F}(q, \omega)=\frac{d}{E_{q}^{(2)}-E_{q}^{(1)}}\left(\delta\left(\omega-E_{q}^{(1)}\right)-\delta\left(\omega-E_{q}^{(2)}\right)\right) .
\end{gathered}
$$

The spectral functions (28) - (30) obey the following sum rules:

$$
\begin{gathered}
\int_{-\infty}^{+\infty} d \omega B_{\alpha \beta}^{M F}(q, \omega)=I_{\alpha \beta}, \\
\int_{-\infty}^{+\infty} \omega d \omega B_{\alpha \beta}^{M F}(q, \omega)=\Omega_{q}^{\alpha \beta}=\left\langle\left[\imath \dot{A}_{q}, A_{q}^{+}\right]\right\rangle_{\alpha \beta},
\end{gathered}
$$

where the matrices $I_{\alpha \beta}$ and $\Omega_{q}^{\alpha \beta}$ are given by the Eqs.(13), (14).

\section{Self-energy corrections}

The next step is to consider the self-energy corrections to the MF spectrum. Taking into account the self-energy corrections the equation for the spectrum transforms into the following form:

$$
\operatorname{det}\left(\omega \hat{\tau}_{0}-\tilde{\Omega}_{q}-\tilde{\Pi}(q, \omega)\right)=0 .
$$

First we compute the self-energy matrix elements by using mode-coupling approximation in terms of the dressed particle-hole and spin fluctuations (see, e. g., Götze et al., [17]). This scheme is essentially equivalent to the self-consistent Born approximation in which the vertex corrections are neglected. The proposed scheme is defined by the following decoupling of the time-dependent correlation functions:

$$
\begin{aligned}
\left\langle X_{m}^{-0}(t) X_{i}^{0+}(t) X_{j}^{+0} X_{l}^{0-}\right\rangle & \simeq\left\langle X_{m}^{-0}(t) X_{l}^{0-}\right\rangle\left\langle X_{i}^{0+}(t) X_{j}^{+0}\right\rangle, \\
\left\langle\sigma_{i}^{z}(t) S_{m}^{-}(t) \sigma_{j}^{z} S_{l}^{+}\right\rangle & \simeq\left\langle\sigma_{i}^{z}(t) \sigma_{j}^{z}\right\rangle\left\langle S_{m}^{-}(t) S_{l}^{+}\right\rangle \\
\left\langle S_{i}^{z}(t) S_{m}^{-}(t) S_{j}^{z} S_{l}^{+}\right\rangle & \simeq\left\langle S_{i}^{z}(t) S_{j}^{z}\right\rangle\left\langle S_{m}^{-}(t) S_{l}^{+}\right\rangle
\end{aligned}
$$

The self-energy matrix elements are obtained by using the above defined decoupling scheme (33) and (35) with the spectral representation for the GF. The diagonal elements involve two contributions:

$$
\Pi_{11(22)}(q, \omega)=\Pi_{11(22)}^{(1)}(q, \omega)+\Pi_{11(22)}^{(2)}(q, \omega) .
$$

The first one describes fluctuations of the internal degrees of freedom of the given spin subsystem. While the second one stems from the Hund's term and describes the coupling between itinerant and core spins.

For the itinerant spins the first term in Eq. (36) is due to decay of spin fluctuations into particle-hole pair excitations and reads as

$$
\Pi_{11}^{(1)}(q, \omega)=\frac{1}{N} \int_{-\infty}^{+\infty} \int_{-\infty}^{+\infty} d \omega^{\prime} d \omega_{1} \frac{n\left(\omega_{1}-\omega^{\prime}\right)-n\left(\omega_{1}\right)}{\omega-\omega^{\prime}+\imath \varepsilon}
$$




$$
\times \sum_{k, \sigma} t_{k q}^{2} A^{\sigma}\left(k-q, \omega_{1}-\omega^{\prime}\right) A^{\bar{\sigma}}\left(k, \omega_{1}\right)
$$

where $t_{k q}=z t\left(\gamma_{k}-\gamma_{k-q}\right), n(\omega)=\left(e^{\beta \omega}+1\right)^{-1}$, and $A^{\sigma}(k, \omega)$ is the single-electron spectral function. By using the MF approximation for that, Eq. (A.7), we can integrate over the frequencies in Eq. (37) and obtain the following estimation:

$$
\Pi_{11}^{(1)}(q, \omega)=\frac{1}{N} \sum_{k, \sigma} t_{k q}^{2}\left(1-n^{\sigma}\right)\left(1-n^{\bar{\sigma}}\right) \frac{n\left(\varepsilon_{k-q}^{\sigma}\right)-n\left(\varepsilon_{k}^{\bar{\sigma}}\right)}{\omega+\varepsilon_{k-q}^{\sigma}-\varepsilon_{k}^{\bar{\sigma}}+\imath \varepsilon} .
$$

It has the standard form for a one-loop particle-hole contribution to the self-energy (see, e.g. 20]).

The second terms in Eq.(36) are the same for both subsystem and coincides with the nondiagonal elements of the self-energy matrix due to the Hund coupling

$$
\Pi_{11(22)}^{(2)}(q, \omega)=-\Pi_{12(21)}(q, \omega)=\Pi_{H}(q, \omega)
$$

with

$$
\begin{array}{r}
\Pi_{H}(q, \omega)=\left(\frac{J_{H}}{2 S}\right)^{2} \frac{1}{N \pi^{2}} \int_{-\infty}^{+\infty} \int_{-\infty}^{+\infty} d \omega^{\prime} d \omega_{1} \frac{1+N\left(\omega^{\prime}-\omega_{1}\right)+N\left(\omega_{1}\right)}{\omega-\omega^{\prime}+\imath \varepsilon} \\
\times \sum_{k}\left[\operatorname{Im} \chi_{22}^{z}\left(k, \omega_{1}\right) \operatorname{Im} \chi_{11}\left(k-q, \omega^{\prime}-\omega_{1}\right)+\operatorname{Im} \chi_{22}\left(k, \omega_{1}\right) \operatorname{Im} \chi_{11}^{z}\left(k-q, \omega^{\prime}-\omega_{1}\right)\right]
\end{array}
$$

where $N(\omega)=\left(e^{\beta \omega}-1\right)^{-1}, \chi_{11}^{z}$ and $\chi_{22}^{z}$ denotes the longitudinal susceptibility of the itinerant and core spins, respectively.

Let us consider now the remaining $\Pi_{22}^{(1)}$ term which describes the fluctuations in the core spin subsystem. This contribution is due to the Heisenberg exchange between the localized spins and is given by

$$
\begin{aligned}
\Pi_{22}^{(1)}(q, \omega)=\frac{1}{N \pi^{2}} \int_{-\infty}^{+\infty} \int_{-\infty}^{+\infty} d \omega^{\prime} d \omega_{1} \frac{1+N\left(\omega^{\prime}-\omega_{1}\right)+N\left(\omega_{1}\right)}{\omega-\omega^{\prime}+\imath \varepsilon} \\
\times \sum_{k} J_{k q}^{2} \operatorname{Im} \chi_{22}^{z}\left(k-q, \omega_{1}\right) \operatorname{Im} \chi_{22}\left(k, \omega^{\prime}-\omega_{1}\right)
\end{aligned}
$$

where $J_{k q}=z J\left(\gamma_{k}-\gamma_{k-q}\right)$.

In order to evaluate the longitudinal susceptibility in Eqs. (40), (41) for both subsystems we will use for them the simplest one-loop approximation (see, e.g. [20]). In this approximation the imaginary part of $\chi_{11}^{z}(q, \omega)$ is given as the convolution of the single-electron GFs

$$
\begin{gathered}
-\frac{1}{\pi} \operatorname{Im} \chi_{11}^{z}(q, \omega)=\frac{1}{4 N} \int_{-\infty}^{+\infty} d \omega^{\prime}\left[n\left(\omega^{\prime}-\omega\right)-n\left(\omega^{\prime}\right)\right] \\
\times \sum_{k, \sigma} A^{\sigma}\left(k, \omega^{\prime}\right) A^{\sigma}\left(k-q, \omega^{\prime}-\omega\right) .
\end{gathered}
$$

The imaginary part of the core spin susceptibility $\chi_{22}^{z}$ can be expressed in the linear spin-wave approximation as

$$
-\frac{1}{\pi} \operatorname{Im} \chi_{22}^{z}(q, \omega)=\frac{1}{\pi^{2} 4 S^{2} N} \int_{-\infty}^{+\infty} d \omega^{\prime}\left(N\left(\omega^{\prime}-\omega\right)-N\left(\omega^{\prime}\right)\right)
$$




$$
\sum_{k} \operatorname{Im} \chi_{22}\left(k-q, \omega^{\prime}-\omega\right) \operatorname{Im} \chi_{22}\left(k, \omega^{\prime}\right),
$$

which follows directly from the Holstein-Primakoff representation.

To study the spin wave spectrum including self-energy corrections let us consider the static limit for $q \rightarrow 0$. For the self-energy matrix we can write

$$
\lim _{q \rightarrow 0} \Pi(q, 0)=\left(\begin{array}{cc}
-A q^{2}-d_{1} & d_{1} \\
d_{1} & -B q^{2}-d_{1}
\end{array}\right)
$$

where

$$
A=-\lim _{q \rightarrow 0} \frac{\Pi_{11}^{(2)}(q, 0)}{q^{2}}, B=-\lim _{q \rightarrow 0} \frac{\Pi_{22}^{(2)}(q, 0)}{q^{2}}, d_{1}=-\Pi^{J_{H}}(0,0) .
$$

Here the coefficient $A, B$, and $d_{1}$ are positive since $\Pi(q, 0)<0$ in the second order of the perturbation theory. As it follows from Eqs. (44) and (45), the selfenergy corrections coming from the Hund's coupling does not renormalize the spin stiffness and gives input only into the gap. Hence the spin-wave spectrum in the longwavelegth limit can be written as

$$
\begin{aligned}
\omega_{q}^{(1)} \simeq \tilde{D}_{1} q^{2} \\
\omega_{q}^{(2)} \simeq \tilde{\Delta}+\tilde{D}_{2} q^{2}
\end{aligned}
$$

where the renormalized spin stiffness and the gap are given by

$$
\begin{array}{r}
\tilde{D}_{1}=\frac{a-b-A-B}{12\left(\left\langle S^{z}\right\rangle+\left\langle\sigma^{z}\right\rangle\right)} \\
\tilde{D}_{2}=\frac{(a-A)\left\langle S^{z}\right\rangle^{2}-(b+B)\left\langle\sigma^{z}\right\rangle^{2}}{12\left\langle S^{z}\right\rangle\left\langle\sigma^{z}\right\rangle\left(\left\langle S^{z}\right\rangle+\left\langle\sigma^{z}\right\rangle\right)} \\
\tilde{\Delta}=\frac{\left(d-d_{1}\right)\left(\left\langle S^{z}\right\rangle+\left\langle\sigma^{z}\right\rangle\right)}{2\left\langle S^{z}\right\rangle\left\langle\sigma^{z}\right\rangle} .
\end{array}
$$

Now we consider the damping for the acoustic spin wave mode given by the imaginary parts of the self-energy matrix. For the damping induced by particle-hole excitations we get from Eq. (38) in the MF approximation for the single-electron GFs:

$$
\begin{gathered}
\Gamma_{11}^{(1)}(q, \omega)=-\operatorname{Im} \Pi_{11}^{(1)}(q, \omega+\imath \varepsilon) \\
=\frac{\pi}{N} \sum_{k, \sigma} t_{k q}^{2}\left(1-n^{\sigma}\right)\left(1-n^{\bar{\sigma}}\right)\left[n\left(\varepsilon_{k-q}^{\sigma}\right)-n\left(\varepsilon_{k}^{\bar{\sigma}}\right)\right] \delta\left(\omega+\varepsilon_{k-q}^{\sigma}-\varepsilon_{k}^{\bar{\sigma}}\right)
\end{gathered}
$$

The contribution, due to the finite $k$-independent gap in single-electron spectrum in the ferromagnetic state disappears in the low frequency limit, $\Gamma_{11}^{(1)}(q, \omega)=0$ for $\omega<h$ (see Eq. (A.14)).

The damping due to the antiferomagnetic exchange interaction given by the imaginary part of the self-energy $\Pi_{22}^{(1)}(q, \omega)$, Eq. (41), gives a small contribution proportional to $q^{2}$ in the longwavelength limit and can be disregarded due to small antiferromagnetic exchange interaction $J$. 
The largest contribution to the damping of spin waves is given by the imaginary part of the self-energy due to the Hund coupling, Eq. (40):

$$
\begin{aligned}
& \Gamma_{H}(q, \omega)=-\operatorname{Im} \Pi_{H}(q, \omega+\imath \varepsilon)=\left(\frac{J_{H}}{2 S}\right)^{2} \frac{1}{\pi N} \int_{-\infty}^{+\infty} d \omega_{1}\left(1+N\left(\omega-\omega_{1}\right)+N\left(\omega_{1}\right)\right) \\
& \times \sum_{k}\left[\operatorname{Im} \chi_{22}\left(k-q, \omega-\omega_{1}\right) \operatorname{Im} \chi_{11}^{z}\left(k, \omega_{1}\right)+\operatorname{Im} \chi_{11}\left(k-q, \omega-\omega_{1}\right) \operatorname{Im} \chi_{22}^{z}\left(k, \omega_{1}\right)\right] .
\end{aligned}
$$

Here for the imaginary parts of the spin susceptibilities $\chi_{11}(k, \omega)$ and $\chi_{22}(k, \omega)$ we will use their MF values in Eqs. (28), (29) taking into account only the acoustic $E_{q}^{(1)}$ mode:

$$
\begin{aligned}
& -\frac{1}{\pi} \operatorname{Im} \chi_{11}^{M F}(q, \omega) \simeq 2\left\langle\sigma^{z}\right\rangle \frac{\tilde{\Omega}_{q}^{22}-E_{q}^{(1)}}{E_{q}^{(2)}-E_{q}^{(1)}} \delta\left(\omega-E_{q}^{(1)}\right)=\Lambda_{q}^{11} \delta\left(\omega-E_{q}\right) \\
& -\frac{1}{\pi} \operatorname{Im} \chi_{22}^{M F}(q, \omega) \simeq 2\left\langle S^{z}\right\rangle \frac{\tilde{\Omega}_{q}^{11}-E_{q}^{(1)}}{E_{q}^{(2)}-E_{q}^{(1)}} \delta\left(\omega-E_{q}^{(1)}\right)=\Lambda_{q}^{22} \delta\left(\omega-E_{q}\right) .
\end{aligned}
$$

For the longitudinal spin susceptibilities in (49) we will use Eqs. (42), (43).

After integration over $\omega_{1}$ we get the following result

$$
\begin{gathered}
\Gamma_{H}(q, \omega)=\left(e^{\omega / T}-1\right)\left(\frac{J_{H}}{2 S}\right)^{2} \frac{\pi}{4 N^{2}} \sum_{k, k_{1}, \sigma} \Lambda_{k-q}^{22}\left(1-n_{\sigma}\right)^{2} \\
\times N\left(E_{k-q}\right) n\left(\varepsilon_{k_{1}}^{\sigma}\right)\left[1-n\left(\varepsilon_{k_{1}-k}^{\sigma}\right)\right] \delta\left(\omega-E_{k-q}+\varepsilon_{k_{1}-k}^{\sigma}-\varepsilon_{k_{1}}^{\sigma}\right) \\
+\left(e^{\omega / T}-1\right)\left(\frac{J_{H}}{2 S}\right)^{2} \frac{\pi}{4 S^{2} N^{2}} \sum_{k, k_{1}} \Lambda_{k-q}^{11} \Lambda_{k_{1}}^{22} \Lambda_{k_{1}-k}^{22} \\
\times N\left(E_{k-q}\right) N\left(E_{k_{1}}\right)\left[1+N\left(E_{k_{1}-k}\right)\right] \delta\left(\omega-E_{k-q}^{(1)}+E_{k_{1}-k}^{(1)}-E_{k_{1}}\right) .
\end{gathered}
$$

It describes a spin wave damping due to its decay into an electron-hole pair and another spin wave (the first term) and a three spin-wave scattering process (the second term). The latter has a standard form for three magnon scattering (see, $(31.2 .20)$ in [21]). At low energy regime $(\omega \rightarrow 0)$ and at the longwavelenght limit the requirements for conservation of energy and momentum allow only small wave vectors and thus only small energies. Hence we can consider $\Lambda_{k}^{11}$ and $\Lambda_{k}^{22}$ as $k$ independent. In the limit $k \rightarrow 0$ we obtain

$$
\Lambda_{k}^{11} \simeq \Lambda_{0}^{11}=2\left\langle\sigma^{z}\right\rangle \frac{\left\langle\sigma^{z}\right\rangle}{\left\langle S^{z}\right\rangle+\left\langle\sigma^{z}\right\rangle}, \Lambda_{k}^{22} \simeq \Lambda_{0}^{11}=2\left\langle S^{z}\right\rangle \frac{\left\langle S^{z}\right\rangle}{\left\langle S^{z}\right\rangle+\left\langle\sigma^{z}\right\rangle}
$$

In this approximation from the Eq. (52) follows that at low energies, $(\omega \ll T)$, the damping has a linear $\omega$-dependence, $\Gamma^{J_{H}}(q, \omega) \sim \omega$, and does not depend explicitly on the wave vector $q$. In the low temperature region, $\left(\omega \ll T \ll \omega_{0}\right)$ where $\omega_{0}$ is the maximal acoustic spin-wave frequency, estimations for $\Gamma_{H}(q, \omega) / \omega$ show that the first contribution in Eq. (52) is proportional to $\left(T / \omega_{0}\right)\left(J_{H}^{2} / N\left(\epsilon_{F}\right) v_{F} k_{0}\right)$ where $N\left(\epsilon_{F}\right)$ is the density of state at the Fermi level and $v_{F}$ is the Fermi velocity, $k_{0} \simeq 2 \pi / a$ and $a$ is a lattice constant. The second term gives to the damping the contribution proportional to $\left(T / \omega_{0}\right)\left(J_{H} / \omega_{0}\right)^{2}$. To give more accurate estimations for the spin-wave damping numerical studies should be performed which will be considered elsewhere. 


\section{Conclusions}

In the present paper we have calculated dynamical spin susceptibility for the generalized ferromagnetic Kondo model (1) by taking into account explicitly both the strong Hund interaction for the itinerant $e_{g}$ and localized $t_{2 g}$ electrons and AFM interaction between the $t_{2 g}$ electrons. We consider the ferromagnetic phase and therefore neglect a possible orbital ordering of the $e_{g}$ electrons. Strong electron correlations between $e_{g}$ electrons are treated within the Hubbard operator technique which is important in calculation of the single-electron GF for the itinerant electrons.

We have proved that even in the MFA described by the frequency matrix, Eq. (19), we get the , acoustic spin-wave excitations, Eq. (24), due to coupling of the two modes with gaps for itinerant and localized electrons. The gapless mode should appear in the model (1) with rotation symmetry for spin system. A gapless mode in the limit $J_{H} \rightarrow \infty$, considered in Ref. [14], was obtained only by taking into account self-energy corrections. In our case the self energy corrections calculated in the self-consistent Born approximation, Eqs. (32)-(34), resulted in additional renormalization of the stiffness of the acoustic ferromagnetic spin waves. The imaginary parts of the self-energy gives the damping of spin waves. We have evaluated the most important contribution due to Hund coupling in the second order, Eq. (49), which can be described as a three magnon scattering. The damping of acoustic spin waves is proportional to the frequency for $\omega \ll T$, Eq. (51), and should be small for small wave vectors. To give numerical estimations one should solve self-consistently the system of equations for the matrix spin susceptibility, Eq. (6), and the self-energy functions, Eq. (37). Also the spectrum of single electron excitations, given in the Appendix, should be evaluated to consider the itinerant electron contributions to the spin waves, Eq. (36). These problems will be considered elsewhere.

\section{Acknowledgments}

We wish to thank G.Jackeli for valuable discussions. A partial financial support by the INTAS Program, Grant No 97-0963, is acknowledged.

\section{Appendix}

In this Appendix we evaluate the single-electron Green function defined as

$$
G^{\sigma}(k, \omega)=\left\langle\left\langle X_{k}^{0 \sigma} \mid X_{k}^{\sigma 0}\right\rangle\right\rangle_{\omega}
$$

with the corresponding spectral function $A_{k}^{\sigma}(\omega)=-(1 / \pi) \operatorname{Im} G^{\sigma}(k, \omega+\imath \varepsilon)$. In the site representation the equation of motion for $G^{\sigma}(k, \omega)$ reads as

$$
\omega\left\langle\left\langle X_{i}^{0 \sigma} \mid X_{k}^{\sigma 0}\right\rangle\right\rangle_{\omega}=\left\langle\left\{X_{i}^{0 \sigma}, X_{j}^{\sigma 0}\right\}\right\rangle+\left\langle\left\langle\imath \dot{X_{i}^{0 \sigma}} \mid X_{j}^{\sigma 0}\right\rangle\right\rangle_{\omega}
$$

Using the commutation relations, Eq.(幽, we obtain

$$
\imath \dot{X_{i}^{0 \sigma}}=\left[X_{i}^{0 \sigma}, H\right]=-\sum_{l \neq i} t_{i l}\left[\left(X_{i}^{00}+X_{i}^{\sigma \sigma}\right) X_{l}^{0 \sigma}+X_{i}^{\bar{\sigma} \sigma} X_{l}^{0 \bar{\sigma}}\right]
$$




$$
-\frac{J_{H}}{4 S}\left[\sigma S_{i}^{z} X_{i}^{0 \sigma}+S_{i}^{\bar{\sigma}} X_{i}^{0 \bar{\sigma}}\right]
$$

The next step is to define the irreducible part $Z_{i}^{0 \sigma}$ of the current operator $\imath \dot{X_{i}^{0 \sigma}}$ by

$$
\imath \dot{X_{i}^{0 \sigma}}=\sum_{l} \varepsilon_{i l}^{\sigma} X_{l}^{0 \sigma}+Z_{i}^{0 \sigma},\left\langle\left\{Z_{i}^{0 \sigma}, X_{j}^{\sigma 0}\right\}\right\rangle=0 .
$$

The definition gives for the frequency matrix

$$
\varepsilon_{i j}^{\sigma}=\left\langle\left\{\imath \dot{X}_{i}^{0 \sigma}, X_{j}^{\sigma 0}\right\}\right\rangle /\left(1-n^{\bar{\sigma}}\right),
$$

where by using the completeness relation, Eq.(2), we write $\left\langle\left\{X_{i}^{0 \sigma}, X_{j}^{\sigma 0}\right\}\right\rangle=\delta_{i, j}(1-$ $n^{\bar{\sigma}}$ ) with $n^{\bar{\sigma}}=\left\langle n_{i}^{\bar{\sigma}}\right\rangle$. By using equation of motion (A.3) we get

$$
\begin{array}{r}
\left\langle\left\{\imath \dot{X}_{i}^{0 \sigma}, X_{j}^{\sigma 0}\right\}\right\rangle=-t_{i j}\left\langle\left(1-n_{i}^{\bar{\sigma}}\right)\left(1-n_{j}^{\bar{\sigma}}\right)\right\rangle-t_{i j}\left\langle X_{i}^{\bar{\sigma} \sigma} X_{j}^{\sigma \bar{\sigma}}\right\rangle \\
+\sum_{l} t_{i l}\left\langle X_{i}^{\bar{\sigma} 0} X_{l}^{0 \bar{\sigma}}\right\rangle-\frac{J_{H}}{4 S} \sigma\left\langle S_{i}^{z}\right\rangle\left(1-n^{\bar{\sigma}}\right) \delta_{i j}-\frac{J_{H}}{4 S}\left\langle S_{i}^{\bar{\sigma}} X_{i}^{\sigma \bar{\sigma}}\right\rangle \delta_{i j} .
\end{array}
$$

In the present paper we will not include the self-energy correction coming from $Z_{i}^{0 \sigma}$ term (A.4) and treat the single-electron GF within the linear, MF type approximation. That results in the following form of the single-electron GF

$$
G^{\sigma}(k, \omega)=\frac{1-n^{\bar{\sigma}}}{\omega-\varepsilon_{k}^{\sigma}} .
$$

By introducing the nearest neighbor charge-spin correlation function

$$
\begin{aligned}
N_{1, \sigma}^{c s}= & \frac{1}{N} \sum_{k} \gamma_{k}\left[\left\langle X_{k}^{\bar{\sigma} \bar{\sigma}} X_{-k}^{\bar{\sigma} \bar{\sigma}}\right\rangle+\left\langle X_{k}^{\bar{\sigma} \sigma} X_{k}^{\sigma \bar{\sigma}}\right\rangle\right] \\
& =\left\langle X_{i}^{\bar{\sigma} \bar{\sigma}} X_{i+a}^{\bar{\sigma} \bar{\sigma}}\right\rangle+\left\langle X_{i}^{\bar{\sigma} \sigma} X_{i+a}^{\sigma \bar{\sigma}}\right\rangle
\end{aligned}
$$

we can rewrite the frequency matrix in the form

$$
\varepsilon_{i j}^{\sigma}=\epsilon^{\sigma} \delta_{i j}+\epsilon_{i j}^{\sigma} .
$$

where

$$
\epsilon_{i j}^{\sigma}=t_{i j}\left[\left(1-2 n^{\bar{\sigma}}\right)+N_{1, \sigma}^{c s}\right] /\left(1-n^{\bar{\sigma}}\right)
$$

is the one particle spectrum in the linear approximation, and

$$
\epsilon^{\sigma}=-\frac{J_{H}}{4 S}\left[\sigma\left\langle S^{z}\right\rangle+\frac{\left\langle S_{i}^{\bar{\sigma}} X_{i}^{\sigma \bar{\sigma}}\right\rangle}{1-n^{\bar{\sigma}}}\right]+\frac{z t n_{1}^{\bar{\sigma}}}{1-n^{\bar{\sigma}}}
$$

is the spin- dependent energy shift of the spectrum. In the momentum space the spectrum is

$$
\varepsilon_{k}^{\sigma}=\sum_{R_{i j}} e^{-\imath k R_{i j}} \varepsilon_{i j}^{\sigma}=\epsilon^{\sigma}-z t_{e f f}^{\sigma} \gamma_{k}
$$

where

$$
t_{\text {eff }}^{\sigma}=t\left[\left(1-2 n^{\bar{\sigma}}\right)+N_{1, \sigma}^{c s}\right] /\left(1-n^{\bar{\sigma}}\right),
$$

is an effective bandwidth that is narrowed and spin dependent due to the spin and charge correlations. In the MFA in the ferromagnetic state we have a spin gap in the single-electron spectrum:

$$
\varepsilon_{k}^{\bar{\sigma}}-\varepsilon_{k}^{\sigma} \simeq \sigma\left(J_{H} / 2 S\right)\left\langle S^{z}\right\rangle=\sigma h .
$$




\section{References}

[1] A.P.Ramirez, J. Phys. Cond. Matter 9, 8171, (1997).

[2] A.J.Millis et al., Phys. Rev. B 54, 5389 and 5405, (1996).

[3] D.I.Khomskii, G.A.Sawatzky, Sol.St.Comm.102, 87, (1997).

[4] J.B.Goodenough, Magnetism and Chemical Bonds, Intersc. publ., NY, (1963).

[5] C. Zener, Phys.Rev. 82, 403, (1951)

[6] P.W.Anderson, H.Hasegawa, Phys.Rev. 100, 675, (1955).

[7] P.G. de Gennes, Phys.Rev. 118, 141, (1960).

[8] P.Schieffer et al., Phys. Rev. Lett. 75, 3396, (1995).

[9] J.Inoue, S.Maekawa, Phys. Rev. Lett. 74, 3407, (1995).

[10] S. Ishihara, J.Inoue, S.Maekawa, Phys.Rev.B 55, 8280, (1997).

[11] A.M.Balagurov et al., JETP Lett., to be published.

[12] T.G.Perring et al., Phys. Rev. Lett. 78, 3197, (1997).

[13] J.W.Lynn et al., Phys. Rev. Lett. 76, 4046, (1996).

[14] N.Furukawa, J. Phys. Soc. Jap. 63, 3214, (1994).

[15] L.F.Feiner and A.M.Oles, Phys. Rev. B 56, in press (1998).

[16] S.Satpathy, Z.S.Popovic, F.R.Vukajlovic, Phys.Rev.Lett. 76, 960, (1996).

[17] W. Gotse and P.Wolfle, J. Low Temp. Phys. 5, 575 (1971).

[18] D. N. Zubarev, Usp. Fiz. Nauk. 71, 71, (1960).

[19] Yu. A. Tserkovnikov, Theor. Math. Fiz. 49, 219, (1981); 52, 147, (1982).

[20] Yu.A. Izyumov, M.I. Katsnelson, and Yu.N. Skriabin, Magnetism of itinerant electrons, (Moscow, Nauka) (1994).

[21] A.I.Axiezer, V.G.Bariyaxtar, S.V.Peletminskii, Spin waves, (Moscow, Nauka) (1967). 